\title{
Anti-Inflammatory Role of Cannabidiol and O-1602 in Cerulein-Induced Acute Pancreatitis in Mice
}

\author{
Kun Li, PhD, * Jia-yan Feng, MD, * Yong-yu Li, MD, * Birol Yuece, MD, † Xu-hong Lin, MD, * \\ Liang-ying Yu, MD, * Yan-na Li, MD, * Ya-jing Feng, MD, * and Martin Storr, MD $\dagger$
}

Objectives: The anti-inflammatory effects of O-1602 and cannabidiol (CBD), the ligands of G protein-coupled receptor 55 (GPR55), on experimental acute pancreatitis (AP) were investigated.

Methods: Acute pancreatitis was induced in C57BL mice by intraperitoneal injection of $50 \mu \mathrm{g} / \mathrm{kg}$ cerulein hourly, with a total of 6 times. Drugs $(\mathrm{O}-1602,10 \mathrm{mg} / \mathrm{kg}$, or CBD, $0.5 \mathrm{mg} / \mathrm{kg})$ were given by intraperitoneal injection 2 times at 30 minutes before the first injection and immediately before the fifth cerulein injection. At 3 hours after the last injection, the blood, the lungs, and the pancreas were harvested for the pancreatic enzyme activity, myeloperoxidase activity, and pro-inflammatory cytokines measurement; and the expressions of GPR55 mRNA and protein in the pancreas were detected.

Results: Cannabidiol or O-1602 treatment significantly improved the pathological changes of mice with AP and decreased the enzyme activities, IL- 6 and tumor necrosis factor $\alpha$ levels, and the myeloperoxidase activities in plasma and in the organ tissues. G protein-coupled receptor $55 \mathrm{mRNA}$ and protein expressed in the pancreatic tissue, and the expressions were decreased in the mice with AP, and either CBD or O-1602 attenuated these changes to a certain extent.

Conclusion: Cannabidiol and O-1602 showed anti-inflammatory effects in mice with AP and improved the expression of GPR55 in the pancreatic tissue as well.

Key Words: acute pancreatitis, O-1602, cannabidiol, GPR55

(Pancreas 2013;42: 123-129)

A cute pancreatitis (AP) is an inflammatory disease of pancreas, and it has a potential high morbidity and mortality. The severity of AP can vary from pancreatic edema with slight pain to pancreatic necrosis and hemorrhage with multisystem organ failure, systemic inflammatory response syndrome, and sepsis. ${ }^{1,2}$ Uncontrolled activation of proteolytic enzymes and followed inflammatory response are considered as important factors in the pathogenesis and development of AP. The detection of cytokines such as interleukin 6 (IL-6) and tumor necrosis factor $\alpha(\mathrm{TNF}-\alpha)^{3-5}$ for early assessment and therapeutic targets of AP has been reported intensively.

The anti-inflammatory potential of cannabinoids has been a highly interested topic since the discovery of endocannabinoid

From the *Institute of Digestive Disease, Department of Pathophysiology, Tongji University School of Medicine, Shanghai, China; and †Department of Internal Medicine II, Klinikum Grosshadern, Ludwig Maximilians University, Munich, Germany.

Received for publication December 3, 2011; accepted April 5, 2012.

Reprints: Yong-yu Li, MD, Institute of Digestive Disease, Department of

Pathophysiology, Tongji University School of Medicine, 1239 Siping Rd, 200092 Shanghai, China (e-mail: liyongyu@tongji.edu.cn), and Martin Storr, MD, Department of Internal Medicine II, Klinikum Grosshadern, Ludwig Maximilians University, Marchioninistr 15, 81377 Munich, Germany (e-mail: gidoc@gmx.com).

This work was supported by grants from the National Science Foundation of China to Dr. Yong-yu Li (No. 30971168 and 81141050) and from the Deutsche Forschungsgemeinschaft (DFG) to Dr Martin Storr (STO645/6-1) The authors declare no conflict of interest.

Copyright $(2012$ by Lippincott Williams \& Wilkins system in mammalian. The endocannabinoid system consists of endogenous cannabinoid receptors, the endogenous ligands, and the related synthesizing and degrading enzymes. ${ }^{6,7}$ Evidences show that cannabinoids participate in inflammatory regulation and may become new therapeutic medicines in treating inflammatory diseases. ${ }^{8-10}$ There are clinical reports of cannabisinduced $\mathrm{AP},{ }^{11,12}$ but experimental reports confirm the roles of the 2 "classic" cannabinoid receptors, cannabinoid type 1 (CB1) receptor and cannabinoid type 2 (CB2) receptor in ameliorating pain and the modulation of AP. ${ }^{13-17}$ It was shown that preadministered HU210, a synthetic specific CB1 agonist, protects against cerulein-induced mouse pancreatitis; otherwise, CB1 antagonist rimonabant attenuated the severity of AP in obese mice. ${ }^{14,15}$ One of the endogenous cannabinoids, anandamide, when administered before the induction of AP, aggravated pancreatic damage and administered after the induction of AP, reduced the severity of AP, according to the experimental results of Dembinski et al ${ }^{16}$; whereas Petrella et $\mathrm{al}^{17}$ got the contrary results in which pretreatment with anandamide before the induction of AP worsened the inflammation, but posttreatment improved the course of the disease.

An orphan G protein-coupled receptor 55 (GPR55) found in 1999 is supported as a novel CB receptor, which acts as a non$\mathrm{CB} 1$, non-CB2 receptor, or suggested as cannabinoid "type 3" receptor. ${ }^{18-20}$ Expression and role of GPR55 in AP are far from elucidated. Phytocannabinoid-cannabidiol (CBD) is an important bioactive component of Cannabis sativa without psychotropic effect and is shown acting as GPR55 antagonist without binding to $\mathrm{CB} 1$ and $\mathrm{CB} 2$ receptors. ${ }^{20-22}$ The atypical synthetic cannabinoid O-1602 is an analog of CBD and is identified to bind strongly to GPR 55 with neglect binding capacity to CB1 or CB2 receptor and acts as a GPR55 agonist. ${ }^{23-25}$ In the present study, based on detection of GPR55 in mouse pancreatic tissue, an AP mouse model induced by cerulein was treated with CBD or O-1602. By evaluating the changes of pancreatic histopathology, levels of pro-inflammatory cytokines such as IL-6 and TNF- $\alpha$ in plasma and in pancreas, and myeloperoxidase (MPO) activity in lungs and pancreas, and the changes of GPR55 mRNA and protein expressions in mouse pancreas, the possible effects and mechanisms of the new cannabinoid drugs and their receptor GPR55 were explored.

\section{MATERIALS AND METHODS}

\section{Animals}

At least 60 adult C57BL mice (weighting 22-26 g) were obtained from Shanghai Experimental Animal Center, Academia Sinica. Mice were at a 12:12-hour light-dark cycle in sawdustcoated plastic cages and accessed to standard laboratory chow and tap water ad libitum at a constant temperature of $23^{\circ} \mathrm{C} \pm$ $2{ }^{\circ} \mathrm{C}$ and relative humidity of approximately $60 \%$. The animals were fasted for 12 hours before the experiment and were divided at random into 6 groups with 8 to 10 mice in every group: normal saline (NS) group, CBD group (CBD+NS), O-1602 group (O-1602+NS), cerulein-induced AP group (AP), CBD-treated 
AP group (CBD+AP), and O-1602-treated AP group (O-1602+ AP). All study procedures for animals were complied with international guidelines for the care and use of laboratory animals and approved by the Animal Ethics Committee of Tongji University, Shanghai, China.

\section{Pancreatitis Induction}

The mouse AP model was replicated according to the method described previously. ${ }^{26}$ In brief, mice in the AP group received hourly intraperitoneal (IP) injections of $50-\mu \mathrm{g} / \mathrm{kg}$ cerulein (Sigma Aldrich, Taufkirchen, Germany) for a total of 6 times. In the $\mathrm{CBD}+\mathrm{AP}$ and $\mathrm{O}-1602+\mathrm{AP}$ groups, mice got cerulein with the same procedures as that in the AP group, and 2 additional IP injections with $0.5-\mathrm{mg} / \mathrm{kg} \mathrm{CBD}$ or $10-\mathrm{mg} / \mathrm{kg}$ O-1602 (Biotrend $\mathrm{GmbH}$, Cologne, Germany) were given at 30 minutes before the first and immediately before the fifth cerulein injection. For control groups of NS, O-1602+NS, and CBD+NS groups, mice were given isotonic sodium chloride solution instead of cerulein injection, with the same procedures as that in the APrelated groups.

\section{Samples Collection and Preparation}

At 3 hours after the final injection of cerulein or NS, all animals were euthanized by decapitation under isoflurane anesthesia. The blood was collected in heparin-treated tubes; and the pancreas and lungs were carefully dissected, rinsed with isotonic sodium chloride solution, then weighted and divided into several portions and frozen in liquid nitrogen and stored at $-80{ }^{\circ} \mathrm{C}$ until usage, except a portion of the pancreas was fixed in $10 \%$ formalin. The blood samples were centrifuged immediately at $3000 \mathrm{rpm}$ for 10 minutes, and the samples of plasma were put in tubes and stored at $-80^{\circ} \mathrm{C}$ for later analysis.

\section{Histopathologic Evaluation}

Pancreas fixed in $10 \%$ formalin as previously mentioned was paraffin embedded, sliced $(5 \mu \mathrm{m})$, and stained by hematoxylin and eosin. The slides were studied under a light microscope. Fifteen randomly chosen microscopic fields from 3 slides of 3 mice in every group were examined by an investigator blinded to the protocol and the histopathologic score referred to reports ${ }^{27,28}$ : (1) interstitial and acinar edema: zero, absent; 1 , focal expansion of interlobular septa; 2 , expanded expansion of interlobular septa; 3, focal expansion of intralobular septa; 4, expanded expansion of intralobular septa; (2) presence of vacuolization: zero, absent; 1 , less than $20 \% ; 2,20 \%$ to $35 \%$; 3 , $35 \%$ to $50 \%$; 4 , more than $50 \%$; (3) interstitial infiltration of neutrophils or lymphocytes: zero, absent; 1,1 to 10 /high-power field (HP); 2, 11 to $20 / \mathrm{HP} ; 3,20$ to $30 / \mathrm{HP}$; , more than $30 / \mathrm{HP}$; (4) necrosis of acinar cell: zero, absent; 1,1 to $5 / \mathrm{HP} ; 2,5$ to $10 / \mathrm{HP}$; 3,11 to $15 / \mathrm{HP}$; and 4 , more than $15 / \mathrm{HP}$. In each group, means of the scores in edema, vacuolization, infiltration, and necrosis were presented as results of the final pathological scores.

\section{Amylase and Lipase Activities Detection}

Amylase and lipase activities in plasma were measured by adaptable assay kits (Jianchen, Nanjin, China) and performed according to the manufacturer's instructions. One unit of amylase activity was defined as degrading $10 \mathrm{mg}$ of amylum in 30 minutes at $37^{\circ} \mathrm{C}$. One unit of lipase activity was defined as degrading $1 \mu \mathrm{mol}$ of triglyceride per minute at $37^{\circ} \mathrm{C}$. Result of amylase activity was presented as units per $100 \mathrm{~mL}$ of plasma $(\mathrm{U} / \mathrm{mL})$, and lipase activity was presented as units per liter of plasma $(\mathrm{U} / \mathrm{L})$.

\section{IL-6 and TNF- $\alpha$ Measurement}

Commercial available enzyme-linked immunosorbent assay kits (R\&D Systems, Abingdon, UK) were used to detect the levels of IL- 6 and TNF- $\alpha$ in plasma or pancreatic tissues. The tissues were homogenized with a Pro 200 hand-held laboratory homogenizer (Pro Scientific, Conn) at $2400 \mathrm{rpm}$ in ice-cold saline. An aliquot of the homogenate was taken for protein determination with the bicinchoninic acid (BCA) protein assay (Thermo, Rockford, Ill) and the rest of the homogenate, and the samples of plasma were measured for IL- 6 and TNF- $\alpha$, respectively, following the manufacturer's instructions. Each sample was detected in duplicate, and the optical density was determined by a microplate reader (BioTek, Winooski, Vt). IL-6 and TNF- $\alpha$ levels were calculated as picogram per milliliter of plasma $(\mathrm{pg} / \mathrm{mL})$, and according to the BCA protein assay of the pancreatic tissue, IL-6 level in pancreatic tissue was expressed in picogram per milligram protein (pg/mg protein).

\section{MPO Activity Examination}

Myeloperoxidase activities in lungs and pancreas were measured in the study. Tissue samples were homogenized with the Pro 200 homogenizer at $2400 \mathrm{rpm}$ in ice-cold saline. An aliquot of this homogenate was taken for protein determination with the BCA protein assay, and the rest of the homogenate was used for MPO activity examination by a purchased detection kit (Jianchen, Nanjin, China), according to the manufacturer's instructions. One unit of MPO activity was defined as that degrading $1 \mu \mathrm{mol}$ of hydrogen peroxide per minute at $37^{\circ} \mathrm{C}$. According to the BCA protein assay of the pancreatic tissue, MPO activity was calculated as units per milligram protein and presented as percentage of NS control group at last.

\section{Real-Time Quantitative RT-PCR}

RNA was extracted from pancreatic tissues using Trizol reagent (Takara, Shiga, Japan), and a total of $3 \mu \mathrm{g}$ of RNA from each sample was reverse transcripted into first-strand complementary DNA with Superscript TM II RT Kit (Invitrogen, Carlsbad, Calif) according to the manufacturer's manual. Realtime quantitative reverse transcription polymerase chain reaction (PCR) was performed for the detection of the GPR55 mRNA expression using SYBR Primix Ex TaqTM kit (Takara, Shiga, Japan) in FTC 3000 PCR instrument (Maple Ridge, Shanghai, China). Primers and probes were designed and synthesized by Invitrogen. Polymerase chain reaction amplification was carried out with the following profile: "hot star" with an initial denaturing at $95^{\circ} \mathrm{C}$ for 30 seconds and then 40 cycles of 5 seconds at $94^{\circ} \mathrm{C}$ and 31 seconds at $60^{\circ} \mathrm{C}$, with the intron spanning primers of GPR55. The forward primer sequence of GPR55 used here was $5^{\prime}-$ GGACTCATTGGTACTCCTAAGCTGT- $3^{\prime}$ and reverse sequence was 5'-GCAGATCCCAAAGGTCTTCCT-3'. Glyceraldehyde-3phosphate dehydrogenase was as endogenous control to standard the amount of sample in each reaction, with the forward primer sequence 5'-ACCACAGTCCATGCCATCAC-3', and the reverse primer sequence $5^{\prime}$-TCCACCACCCTGTTGCTGTA-3'. Assays were performed in triplicates. The relative quantification value (double $\Delta \mathrm{CT}$ ) was calculated from each sample, and the expression of GPR55 mRNA from the NS group was selected as calibrator and was set to 1 according to a reference. ${ }^{29}$

\section{Western Blotting}

Pancreatic tissues were homogenized, and the lysate was analyzed by Western blotting according to the method described previously. ${ }^{30}$ The primary rabbit anti-GPR55 antibody (Enzo, Plymouth Meeting, Pa) was used here in a 1:500 dilution and the internal control of $\beta$-actin antibody (Sigma, Taufkirchen, Germany) in a 1:1000 dilution. The appropriate IRDye secondary antibody (Rockland, Me) was applied in a 1:5000 dilution and incubated for 1.5 hours at room temperature. Images taken with 
Odyssey 3.0 imaging system (LI-COR, Lincoln, Neb) were analyzed by image analysis system for optical density of the protein bands; and in each group, the GPR55 protein expression was adjusted with the corresponding $\beta$-actin, respectively. The relative expression value of GPR55 was presented as percent of the NS control group.

\section{Immunohistochemistry}

$\mathrm{G}$ protein-coupled receptor 55 protein expression in pancreatic tissue was also detected by immunohistochemistry described previously. ${ }^{31}$ The paraffin sections with pancreatic tissues were dewaxed by regular procedures and dipped in solution of $0.01-\mathrm{mol} / \mathrm{L}$ citric acid for 1 minute at $95^{\circ} \mathrm{C}$, blocked with $5 \%$ goat serum for 30 minutes, and then incubated with rabbit antiGPR55 polyclonal antibody (Cayman Chemical, Ann Arbor, Mich) overnight at $4^{\circ} \mathrm{C}$ at a dilution of 1:20. Sections were then followed by biotin-labeled goat antirabbit IgG for 15 minutes at $37^{\circ} \mathrm{C}$, and horseradish peroxidase-labeled streptavidin (Jinqiao,
Beijing, China) for 30 minutes at $37^{\circ} \mathrm{C}$. Finally, the specimens were stained with diaminobenzidine, nuclear restained with hematoxylin, and photographed by Leica DM 2500 microscope (Leica, Wetzlar, Germany). Images were semi-quantified by Image-pro plus 6.0 (Mediacy, Trenton, NJ). Five nonoverlapping views were analyzed from each slide and repeated in at least 3 slides from 3 different mice in every group. The average optical density represented the positive staining intensity and was expressed as percent of the NS group at last.

\section{Statistical Analysis}

Values were obtained from multiple determinations in 5 or more separate experiments with a minimum of 6 mice, unless mentioned otherwise. Data were presented as mean \pm SEM. Comparisons between 2 sets of data were made by one-way analysis of variance (SPSS 13.0 software) followed by the Bonferroni post hoc test for multiple treatments. $P<0.05$ was considered statistically significant.
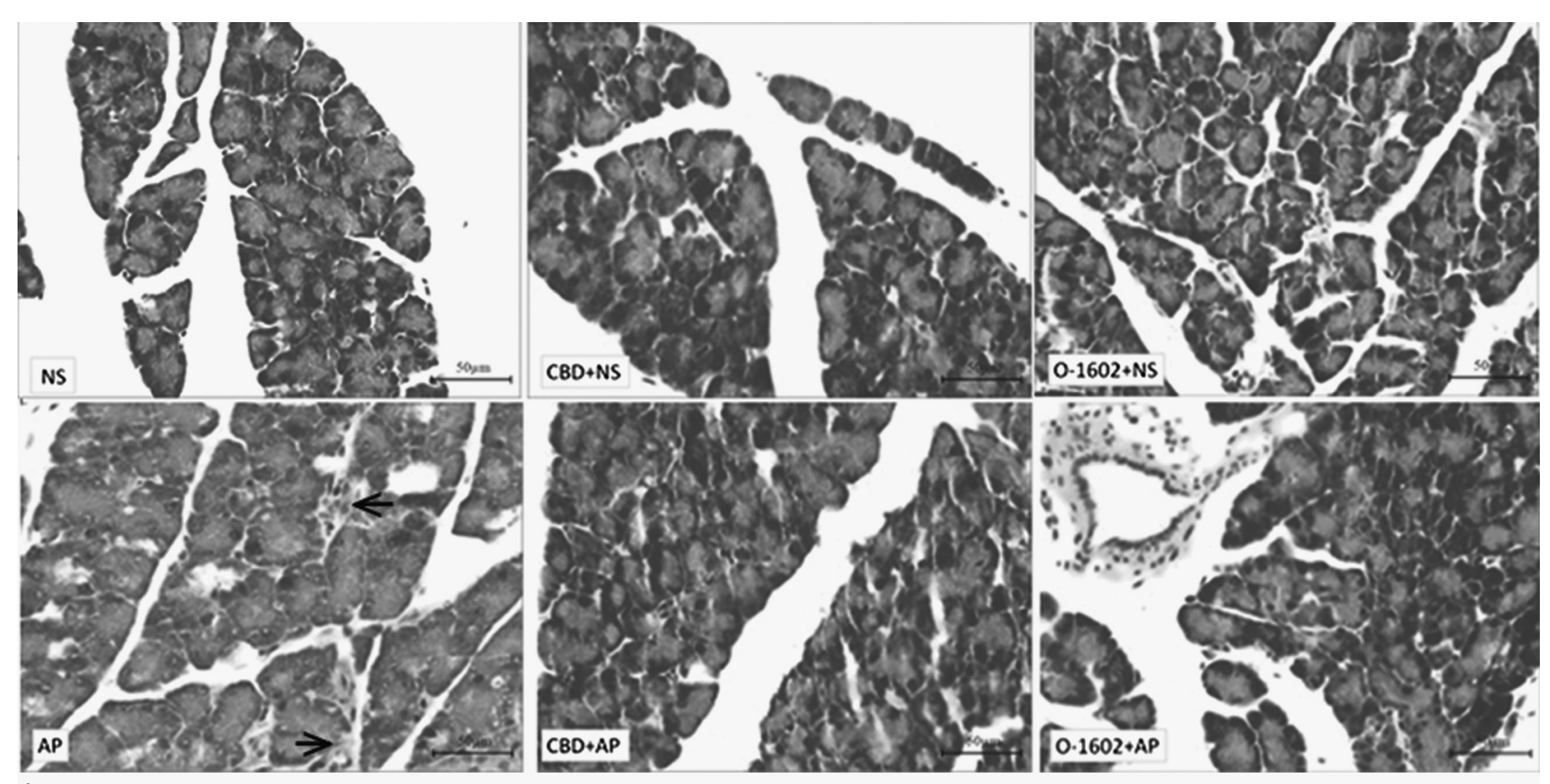

A

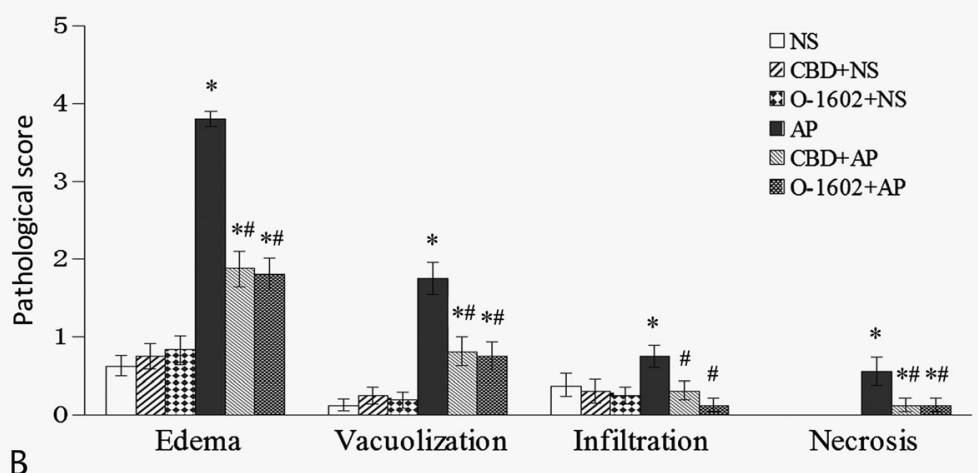

FIGURE 1. Effects of CBD or O-1602 on pancreatic pathology. Pancreatic tissues were harvested from mice at 3 hours after 6 hourly injections of cerulein (AP) or NS and stained by hematoxylin and eosin for microscopic analysis; group CBD+NS and O-1602+NS mice received 2 additional IP injections of $0.5-\mathrm{mg} / \mathrm{kg}$ CBD or $10-\mathrm{mg} / \mathrm{kg}$ O-1602, respectively, based on NS injection; group CBD+AP and

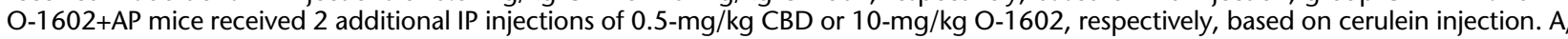
Representative original pathological figures. B, Scores for edema, vacuolization, inflammation, and necrosis in each group. Results are expressed as mean \pm SEM for at least 9 slides from 3 mice in each experimental group. ${ }^{*} P<0.05$ compared with the NS group; ${ }^{\#} P<0.05$ compared with the AP group. Scale bars $=50 \mu \mathrm{m}$. Arrows show the acinous necrosis and/or inflammatory infiltration. 


\section{RESULTS}

\section{Effects of CBD or 0-1602 on Pancreatic Histopathologic Alterations}

Pancreatic tissues in mice treated with cerulein displayed histopathological signs of AP characterized by obvious edema in pancreatic interstices and acinus, acinous vacuolization with slight infiltration, and parenchymal necrosis, as shown in Figure 1A by arrows. Either CBD or O-1602 improved the pathological changes significantly in mice with AP. Cannabidiol or O-1602 alone had no obvious effects on mouse pancreatic histology in the NS control mice (Fig. 1).

\section{Effects of CBD or O-1602 on Amylase and Lipase Activities in Plasma}

Amylase and lipase activities in mouse plasma are presented in Table 1. Compared with the NS control group, cerulein causes nearly 5 times enhancement of amylase activity and 9 times increase of lipase activity, and these elevations were significantly attenuated by either CBD or O-1602 treatment $(P<0.05)$. The administration of CBD (CBD+NS group) or O-1602 alone (O$1602+\mathrm{NS}$ group) had no significant influence on the 2 enzyme activities compared with the NS control group.

\section{Effects of CBD or O-1602 on TNF- $\alpha$ and IL-6 in Plasma or Pancreatic Tissues}

As shown in Figures 2A and B, the levels of TNF- $\alpha$ and IL-6 in plasma were significantly increased in cerulein-induced AP mice compared with those of the NS control mice $(P<0.05)$. Cannabidiol treatment attenuated the increment of TNF- $\alpha$ and IL6 , and O-1602 affected TNF- $\alpha$ level only. In mouse pancreatic tissues, IL-6 level in the AP group was also higher than that in the NS group, and it was attenuated significantly by CBD treatment but not by the administration of O-1602 $(P<0.05$; Fig. $2 \mathrm{C})$. In the $\mathrm{CBD}+\mathrm{NS}$ and $\mathrm{O}-1602+\mathrm{NS}$ groups, $\mathrm{CBD}$ or O-1602 alone had no effects on TNF- $\alpha$ and IL-6 levels in mouse plasma or pancreatic tissues when compared with that of the NS control group.

\section{Effects of CBD or O-1602 on MPO Activity of Lungs and Pancreatic Tissue}

In the NS control group, MPO activity in tissues of lungs and pancreas was $1.19 \pm 0.17$ and $0.58 \pm 0.02 \mathrm{U}$ per gram of protein, respectively, and both of them increased significantly in the mice of the AP group $(P<0.05)$. The treatment of either CBD or O-1602 attenuated the cerulein-induced elevations of MPO activity $(P<0.05)$. Both $\mathrm{CBD}$ and $\mathrm{O}-1602$ alone $(\mathrm{CBD}+\mathrm{NS}$ and $\mathrm{O}-1602+\mathrm{NS}$ groups) had no effects on the MPO activities in the lungs or the pancreas of mice (Figs. 3A, B).

\section{Effects of CBD and 0-1602 on GPR55 mRNA and Protein Expression in Pancreas}

$\mathrm{G}$ protein-coupled receptor 55 mRNA expression in mouse pancreatic tissues was detected by real-time reverse transcription
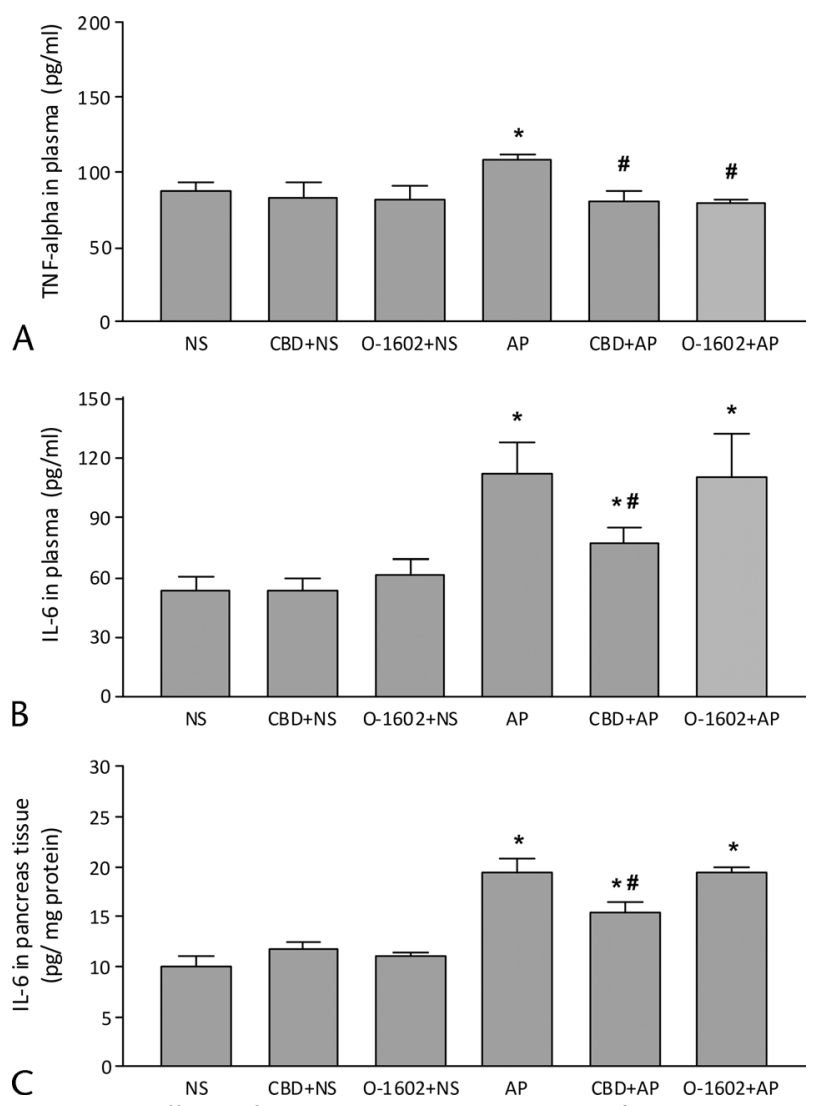

FIGURE 2. Effects of CBD or O-1602 on TNF- $\alpha$ and IL-6. A, TNF- $\alpha$ level in plasma; B, IL-6 level in plasma; C, IL-6 level in pancreas. Assay for TNF- $\alpha$ or IL- 6 was accomplished with enzyme-linked immunosorbent assay kit described in the "Materials and Methods" section. Results are shown as mean \pm SEM; $n=6$ per group. ${ }^{\star} P<0.05$ compared with NS group; ${ }^{\#} P<0.05$ compared with AP group.

PCR, and the semi-quantitative data are shown in Figure 4A. G protein-coupled receptor $55 \mathrm{mRNA}$ expression was decreased markedly in the mice of the AP group when compared with the mice in the NS group $(P<0.05)$, and the reduction was attenuated significantly by either CBD or O-1602 treatment. Nevertheless, CBD or O-1602 treatment had no effects on GPR55 mRNA expression in the pancreatic tissues of the mice in the NS control group.

Western blotting was used to investigate GPR55 protein expression in the pancreatic tissues, and the representative profile of GPR55 protein is shown in Figure 4B. It was discovered that GPR55 was on the site of approximately 37 kilodaltons and $\beta$-actin was on the site of approximately 41 kilodaltons. The

TABLE 1. Effects of CBD or O-1602 on Amylase and Lipase Activities in Plasma

\begin{tabular}{lccccrr}
\hline Group & NS & CBD+NS & O-1602+NS & AP & CBD+AP & O-1602+AP \\
\hline Amylase, U/dL & $719.7 \pm 47$ & $673.1 \pm 39$ & $652.4 \pm 47$ & $3666.4 \pm 99^{*}$ & $2804.2 \pm 114^{\dagger}$ & $2443.0 \pm 112^{\dagger}$ \\
Lipase, U/L & $165.7 \pm 68$ & $192.5 \pm 15$ & $217.2 \pm 29$ & $1315.5 \pm 126^{*}$ & $738.7 \pm 116^{\dagger}$ & $867.7 \pm 113^{\dagger}$ \\
\hline
\end{tabular}

Blood samples were harvested from mice at 3 hours after the last IP injections. Amylase and lipase activities in the plasma were detected by adaptable assay kits as described in the "Materials and Methods". Results are shown as mean \pm SEM; $n=6-8$ per group.

${ }^{*} P<0.05$ compared with the NS group.

${ }^{\dagger} P<0.05$ compared with the AP group. 

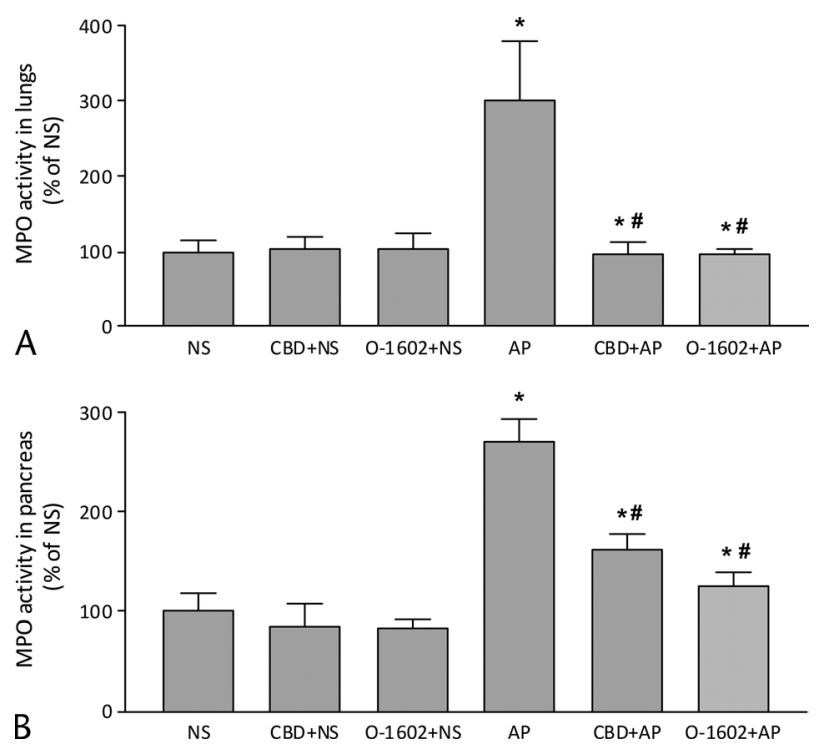

FIGURE 3. Effects of CBD or O-1602 on MPO activity. A, Myeloperoxidase in lungs. $B$, Myeloperoxidase in pancreas. Results are shown as mean \pm SEM; $n=6$ per group. ${ }^{*} p<0.05$ compared with the NS group; ${ }^{\#} P<0.05$ compared with the AP group.

findings showed that the expression of GPR55 protein in the AP group remarkably decreased when compared with that in the NS group $(P<0.05)$, and the reduction was attenuated significantly by either O-1602 or CBD treatment (Fig. 4C). At the same time, CBD or O-1602 treatment had no effects on GPR55 mRNA expression in the NS control group.

\section{Immunohistochemistry Detection of GPR55 Protein in Pancreatic Tissue}

The brownish staining shown in Figure $5 \mathrm{~A}$ represent the expression of GPR55 in the parenchyma of mouse pancreatic tissue. Together with the semi-quantitative analysis of GPR55 expression shown in Figure 5B, it was further demonstrated that in the normal pancreatic tissue, GPR55 protein expressed in a certain amount and seemed not to be affected by CBD or O-1602 alone. However, in the mice of the AP group, there was a decreased GPR55 expression in the pancreatic tissue, and CBD or O-1602 treatment rescued the deduction significantly $(P<0.05)$. The changing trends of GPR55 protein expression detected by Western blotting or immunohistochemistry were highly consistent with that of the GPR55 mRNA expression in the experimental groups.

\section{DISCUSSION}

The incidence of AP has been growing since the 1970s and its treatment is still empiric. ${ }^{32,33}$ Endocannabinoid system has become important targets in many fields in the past 20 years, especially in some inflammatory diseases. Acute pancreatitis is an inflammatory disease of the pancreas, and inflammatory response is important in the pathogenesis and development of the disease. Effects of cannabinoids on AP have been aroused, but the existing studies mainly focused on the classic cannabinoid receptors and showed ambiguous and even paradoxical results. ${ }^{11-17}$

Evidences have pointed that GPR55 is a receptor of the endogenous cannabinoids, and a few of peer-reviewed studies discovered the pharmacology of cannabinoids at GPR55. ${ }^{20,34}$ It has been reported that GPR55 intensively expressed in many kinds of organs in mice, including brain, adrenals, spleen, jejunum and ileum, lung, and kidney, but pancreas has not been mentioned. ${ }^{20}$ Our works discovered that GPR55 is expressed in the pancreatic tissues of mice, as shown in the present study.

Here, we induced mouse AP by IP injection of cerulein, which is used and accepted widely, and the model displayed typical signs of AP in histology and enzymology. Using this model, the early protective roles of the new cannabinoids, O1602 and $\mathrm{CBD},{ }^{20,23-25}$ were explored. From the results, improved pathological changes and decreased lipase and amylase activities were observed, which suggest that both $\mathrm{CBD}$ and $\mathrm{O}-$ 1602 have protective effects on the pancreatic tissues in mice with cerulein-induced AP.

Proinflammatory cytokine burst is involved in the development of AP, and IL- 6 and TNF- $\alpha$ have been proposed as better inflammatory markers of the disease. ${ }^{3-5}$ Plasma levels of IL-6 and TNF- $\alpha$ were detected to make clear the anti-inflammatory roles of O-1602 and CBD. It was demonstrated that the levels of IL-6 and TNF- $\alpha$ in plasma increased significantly in mice with $\mathrm{AP}$, but in the study, CBD could reverse the increased levels of TNF- $\alpha$ and IL- 6 both, whereas O- 1602 reversed the increment of TNF- $\alpha$ only. Then, we checked the level of IL- 6 in the pancreatic tissues of mice with AP, and it was also obviously higher; and CBD, but not O-1602, attenuated the increased levels of IL-6 in the pancreatic tissue of AP mice, the same effective impact as they acted on the plasma level of IL-6. It is known that both IL-6
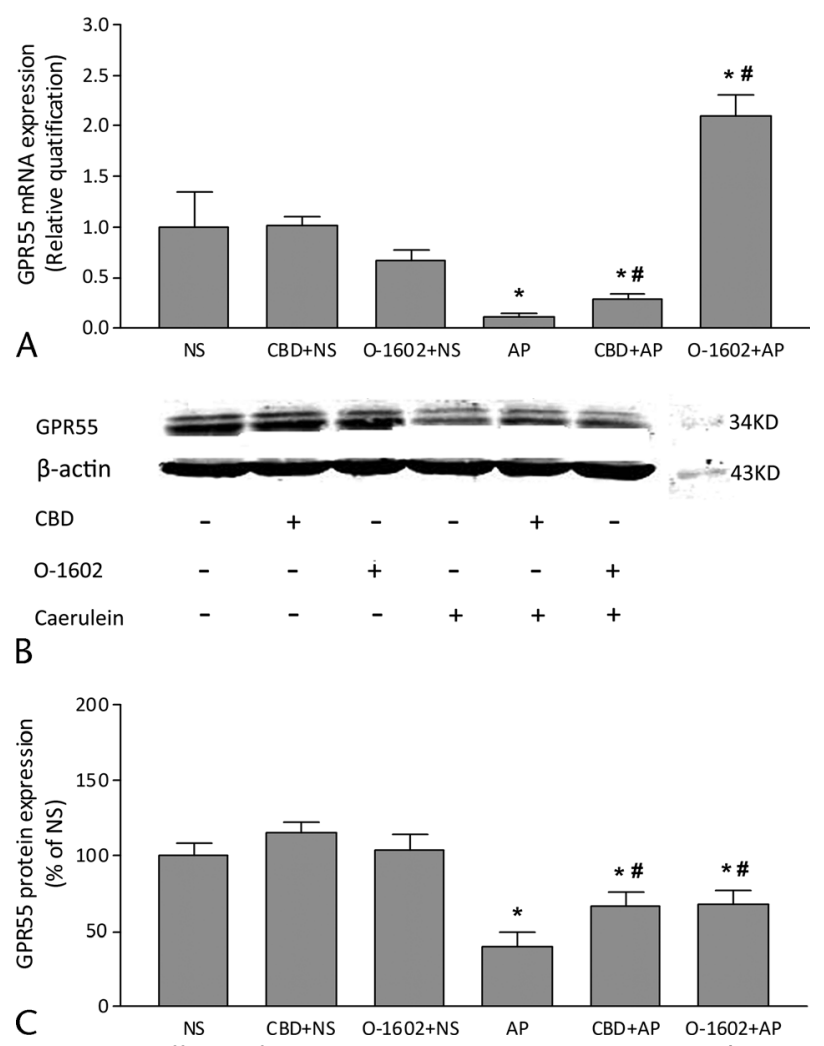

FIGURE 4. Effects of CBD or O-1602 on GPR55 mRNA and protein expression by reverse transcription PCR or Western blotting. A, GPR55 mRNA expression. B, Profiling of GPR55 protein expression by Western blot. C, Densitometric quantification of GPR55 protein expression. Results are shown as mean \pm SEM; $\mathrm{n}=3$ per group. ${ }^{*} P<0.05$ compared with the NS group; ${ }^{\#} P<0.05$ compared with the AP group. 


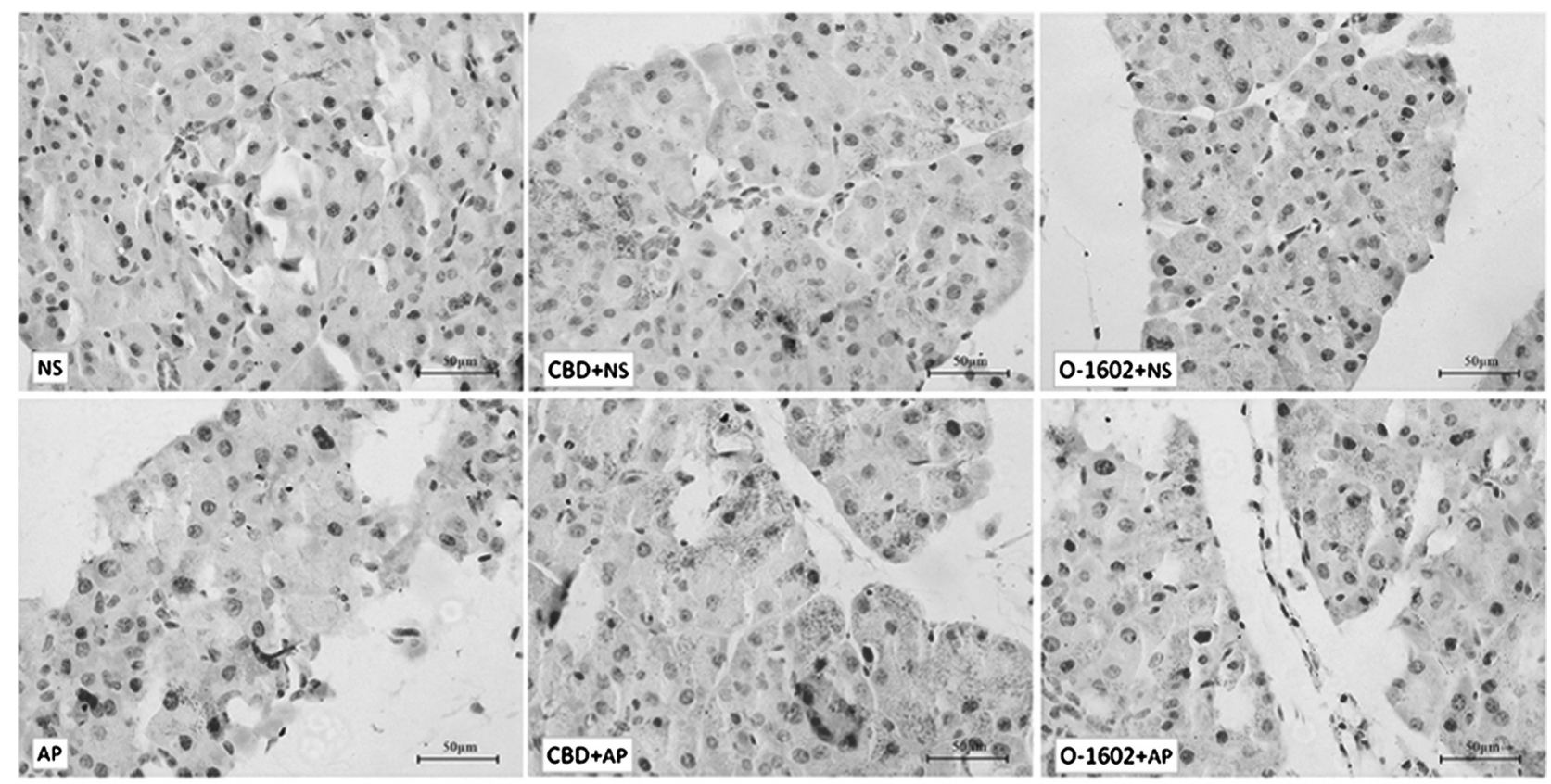

A

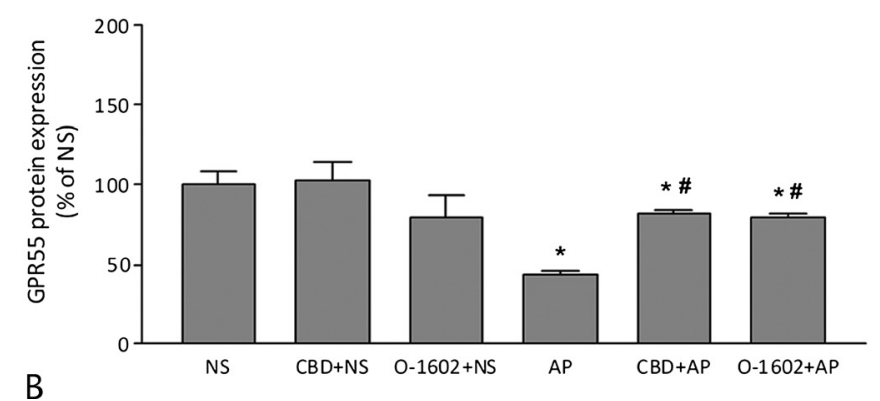

FIGURE 5. Immunohistochemistry detection for GPR55 protein expression. A, Photographed images of GPR55 protein by immunohistochemistry. B, Results of semiquantitative analysis. Five nonoverlapping views were selected from each slide and repeated in at least 9 slides from 3 mice in each experimental group. Results are shown as means \pm SEM. ${ }^{*} P<0.05$ compared with the NS group; ${ }^{\#} P<0.05$ compared with AP group.

and TNF- $\alpha$ are secreted mostly by T cells, macrophages, neutrophils, and endothelial cells; and it has been documented that in AP, pancreatic acinar cells can produce TNF- $\alpha$, and the periacinar myofibroblasts secrete IL-6. ${ }^{35,36}$ According to Granger and Remick, ${ }^{37} \mathrm{TNF}-\alpha$ nicely fits with the model of acinar cell damage progressing to local inflammation, whereas IL-6 is a marker of AP but not a direct mediator of cellular or organ injury. The influence of CBD and O-1602 on pancreatic tissues may be different and leads to their different effects on levels of TNF- $\alpha$ and IL-6. Nevertheless, the anti-inflammatory role of CBD and O-1602 was further demonstrated by their attenuating effects on the increased activity of MPO in lungs and pancreas of mice with AP.

O-1602 is considered an agonist of GPR55, the new putative cannabinoid receptor, whereas CBD is considered an antagonist of it. ${ }^{34}$ However, in the present study, the 2 drugs show weird similar effects on cerulein-induced mouse AP; especially, they showed prevention against the pancreatic damage and the counteract roles on the elevations of the levels of some preinflammatory cytokines, and activities of MPO and pancreatic enzymes, in tissues or in plasma. It has been reported that CBD produces a plethora of pharmacological effects in the body, including antagonizing the $\mathrm{CB} 1$ receptor agonists, inhibiting the activity of fatty acid amide hydrolase, and activating peroxisome proliferator-activated receptor $\gamma$, which take part in the inflammatory modulation in AP. ${ }^{13-17,38-41}$ These results suggest that the anti-inflammatory effects of CBD could work by different mechanisms, except by GPR55-related pathway.

In this study, we found that GPR55 expressed in mouse pancreatic tissue, and both in gene and protein levels. The expression was significantly down-regulated in the pancreas of mice with AP. Cannabidiol or O-1602 reversed the reduction of GPR55 expression to a certain degree. The administration of CBD or O-1602 in normal mice did not affect GPR55 expression. The relationship between the alteration of GPR55 expression in pancreatic tissue and the severity of AP is a valuable concern, which needs further work to clarify.

As previously mentioned, CBD is supposed to act as an antagonist of the GPR55 and O-1602 is a synthetic agonist of the receptor, and both showed a similar protective role and antiinflammatory effect on cerulein-induced mouse AP. To the contradicted results, further investigations are essential to develop to get the detail mechanisms and the reasonable explanations by using specific high selective GPR55 antagonist or GPR55 gene knock-out mice, etc.

On all accounts, the present study discovered that GPR55 exists in mouse pancreatic tissue. Its ligands, O-1602 and CBD, 
have an anti-inflammatory role and protective effect on the pancreatic tissues of mice with cerulein-induced AP, and the new putative cannabinoid receptor GPR55 might be related to the protective effects against AP.

\section{ACKNOWLEDGMENTS}

The authors thank Prof Song Bogen and Ms Zhao Guifeng for the kind help in the pathological examination and evaluation.

\section{REFERENCES}

1. Hochman D, Louie B, Bailey R. Determination of patient quality of life following severe acute pancreatitis. Can J Surg. 2006;49:101-106.

2. Hayden P, Wyncoll D. Severe acute pancreatitis. Curr Anaesth Crit Care. 2008;19:1-7.

3. Bhatia M, Brady M, Shokuhi S, et al. Inflammatory mediators in acute pancreatitis. J Pathol. 2000;190:117-125.

4. Bhatia M. Therapeutic targets for acute pancreatitis and associated multiple organ dysfunction syndrome. Curr Drug Targets Inflamm Allergy. 2002;1:343-351.

5. de-Madaria E, Martinez J, Sempere L, et al. Cytokine genotypes in acute pancreatitis: association with etiology, severity, and cytokine levels in blood. Pancreas. 2008;37:295-301.

6. Pacher P, Batkai S, Kunos G. The endocannabinoid system as an emerging target of pharmacotherapy. Pharmacol Rev. 2006;58:389-462.

7. Faith RK, Nephi S. The therapeutic potential of novel cannabinoid receptors. Pharmacol Ther. 2009;122:83-96.

8. Walter L, Stella N. Cannabinoids and neuroinflammation. $\mathrm{Br} J$ Pharmacol. 2004;141: 775-785.

9. Klein TW. Cannabinoid-based drugs as anti-inflammatory therapeutics. Nat Rev Immunol. 2005;5:400-411.

10. Schicho R, Bashashati M, Bawa M, et al. The atypical cannabinoid O-1602 protects against experimental colitis and inhibits neutrophil recruitments. Inflamm Bowel Dis. 2011;17:1651-1664.

11. Wargo KA, Geveden BN, McConnell V. Cannabinoid-induced pancreatitis: a case series. JOP. 2007;8:579-583.

12. Belze O Jr, Legras A, Ehrmann S, et al. Cannabis-induced acute pancreatitis. J Emerg Med. 2011;131.e3-e4.

13. Michalski CW, Laukert T, Sauliunaite D, et al. Cannabinoids ameliorate pain and reduce disease pathology in cerulein-induced acute pancreatitis. Gastroenterology. 2007;132:1968-1978.

14. Storr M, Kramer J, Michler T, et al. Acute experimental pancreatitis is ameliorated by activation of cannabinoid-1 (CB1) receptor. Gastroenterology. 2008;134:A430.

15. Zyromski NJ, Mathur A, Pitt HA, et al. Cannabinoid receptor-1 blockade attenuates acute pancreatitis in obesity by an adiponectin mediated mechanism. J Gastrointest Surg. 2009;13:831-838.

16. Dembinski A, Warzecha $Z$, Ceranowicz $\mathrm{P}$, et al. Cannabinoids in acute gastric damage and pancreatitis. $J$ Physiol Pharmacol. 2006;57:137-154.

17. Petrella C, Agostini S, Alema GS, et al. Cannabinoid agonist WIN55,212 in vitro inhibits interleukin-6 (IL-6) and monocyte chemo-attractant protein-1 (MCP-1) release by rat pancreatic acini and in vivo induces dual effects on the course of acute pancreatitis. Neurogastroenterol Motil. 2010;22:1248-1256.

18. Sawzdargo M, Nguyen T, Lee DK, et al. Identification and cloning of three novel human $G$ protein-coupled receptor genes GPR52, PsiGPR53 and GPR55: GPR55 is extensively expressed in human brain. Brain Res Mol Brain Res. 1999;64:193-198.

19. Moriconi A, Cerbara I, Maccarrone M, et al. Gpr55: current knowledge and future perspectives of a purported "type 3" cannabinoid receptor. Curr Med Chem. 2010;17:1411-1429.
20. Ryberg E, Larsson N, Sjogren S, et al. The orphan receptor GPR55 is a novel cannabinoid Receptor. Br J Pharmacol. 2007;152: 1092-1101.

21. Pertwee RG. GPR55: a new member of the cannabinoid receptor clan? Br J Pharmacol. 2007;152:984-986.

22. Whytea LS, Rybergb E, Simsc NA, et al. The putative cannabinoid receptor GPR55 affects osteoclast function in vitro and bone mass in vivo. Proc Natl Acad Sci US A. 2009;105:16511-16516.

23. Baker D, Pryce G, Davies WL, et al. In silico patent searching reveals a new cannabinoid receptor. Trends Pharmacol Sci. 2006;27:1-4.

24. Johns DG, Behm DJ, Walker DJ, et al. The novel endocannabinoid receptor GPR55 is activated by atypical cannabinoids but does not mediate their vasodilator effects. Br J Pharmacol. 2007;152:825-831.

25. Mchugh D, Tanner $C$, Mechoulam R, et al. Inhibition of human neutrophil chemotaxis by endogenous cannabinoids and phytocannabinoids: evidence for a site distinct from $\mathrm{CB} 1$ and $\mathrm{CB} 2$. Mol Pharmacol. 2008;73:441-450.

26. Li YY, Ochs S, Gao ZR, et al. Regulation of HSP60 and the role of MK2 in a new model of severe experimental pancreatitis. Am J Physiol Gastrointest Liver Physiol. 2009;297:G981-G989.

27. Cock HE, Forman MA, Farver TB, et al. Prevalence and histopathologic characteristics of pancreatitis in cats. Vet Pathol. 2007;44:39-49.

28. Magana GJ, Lopez CG, Barca A MC. Caerulin-induced pancreatitis in rats: histological and genetic expression changes from acute phase to recuperation. World J Gastroenterol. 2006;12:3999-4003.

29. Breljak D, Ambriovic R, Kapitnovic S, et al. Comparison of three RT-PCR based methods for relative quantification of mRNA. Food Technol Biotechnol. 2005;43:379-388.

30. Li XL, Li K, Li YY, et al. Alteration of Cpn60 expression in pancreatic tissue of rats with acute pancreatitis. Cell Stress Chaperones. 2009;14:199-206

31. Lin XH, Yuece B, Li YY, et al. A novel CB receptor GPR55 and its ligands are involved in regulation of gut movement in rodents. Neurogastroenterol Motil. 2011;23:862-870.

32. Pandol SJ, Saluja AK, Imrie CW, et al. Acute pancreatitis: bench to the bedside. Gastroenterology. 2007;132:1127-1151.

33. Shen HN, Lu CL. Incidence, resource use, and outcome of acute pancreatitis with/without intensive care: a nationwide population-based study in Taiwan. Pancreas. 2011;40:10-15.

34. Ross RA. The enigmatic pharmacology of GPR55. Trends Pharmacol Sci. 2009;30:156-163.

35. Gukovskaya AS, Gukovsky I, Zaninovic V, et al. Pancreatic acinar cells produce, release, and respond to rumor necrosis factor- $\alpha$. Role in regulating cell death and pancreatitis. J Clin Invest. 1997;100: 1853-1862.

36. Shimada M, Andoh A, Hata K, et al. Il-6 secretion by human pancreatic periacinar myofibroblasts in response to inflammatory mediators. J Immunol. 2002;168:861-868.

37. Granger J, Remick D. Acute pancreatitis: models, markers, and mediators. Shock. 2005;24:45-51.

38. Mechoulam R, Peters M, Murillo-Rodriguez E, et al. Cannabidiol recent advances. Chem Biodivers. 2007;4:1678-1692.

39. Ryan D, Drysdale AJ, Pertwee RG, et al. Interactions of cannabidiol with endocannabinoid signalling in hippocampal tissue. Eur J Neurosci. 2007;25:2093-2102.

40. Filippis D, Iuvone T, damico A, et al. Effect of cannabidiol on sepsis-induced motility disturbances in mice: involvement of $\mathrm{CB}$ receptors and fatty acid amide hydrolase. Neurogastroenterol Motil. 2008;20:919-927.

41. O'Sullivan SE, Kendall DA. Cannabinoid activation of peroxisome proliferator-activated receptors: potential for modulation of inflammatory disease. Immunobiology. 2010;215:611-616. 